International Journal Of Occupational Health and Public Health Nursing,

Vol. 6, No. 2, 2020, 75-78

ISSN: 2053-2369 (print version), 2053-2377 (online)

https://doi.org/10.47260/johphn/624

Scientific Press International Limited

\title{
Chronic Kidney Disease in Community
}

\author{
Dimitra Paikopoulou, RN, MSc, PhD $^{1}$
}

\section{Editorial}

The world's disease profile is changing, and chronic diseases now account for the majority of global morbidity and mortality. The causes of chronic kidney diseases (CKD) reflect this change. The increase in prevalence of CKD is partly due to a real increase in its frequency (due to the increase in the average age of people surviving), better detection of CKD, but also to the increasing incidence of diabetes and hypertension among people today, not only within the developed world, but also increasingly within the emerging world ${ }^{(1)}$.

Furthermore, hypertension, smoking, hypercholesterolemia, and obesity, currently among the World Health Organization's (WHO's) top 10 global health risks, are strongly associated with $\mathrm{CKD}^{(2)}$. The factors, together with increasing diabetes prevalence and an aging population, will result in significant global increases in chronic kidney diseases (CKD) end stage renal disease (ESRD) patients ${ }^{(3)}$.

There are approximately 275000 patients with ESRD in the United States and it is estimated that an additional 8 million US adults have kidney disease (defined as a glomerular filtration rate [GFR] of $60 \mathrm{~mL} / \mathrm{min}$ per $1.73 \mathrm{~m} 2)^{(4)}$. Because kidney disease often progresses to ESRD and its attendant complications, the identification of precursors of kidney disease is important, with the belief that interventions will prevent or delay the progression to ESRD.

${ }^{1}$ Laboratory Teaching Staff, Department of Nursing, National and Kapodistrian University of Athens. e-mail:dpaikopoulou@ nurs.uoa.gr

Article Info: Received: September 10, 2020. Revised: September 30, 2020.

Published online: October 1, 2020. 


\section{RISK FACTORS FOR CKD ${ }^{(5-6)}$}

Non-modified risk factors

- Age: loss of the kidneys is a normal part of the aging process, and is most likely due to the progressive atherosclerosis and cardiovascular comorbidity.

- Race: African Americans in the first place and Latin Americans in the second place have proven to be more prone to developing CKD than Caucasian people, in studies in the US.

- Gender: It has been found that end-stage CKD from all causes is more common in men than in women.

- Genetic Factors / Heredity: The likelihood of developing CKD has been linked to family history, even in the absence of proven hereditary kidney disease. Congenital malformations of the urinary tract are one of the major causes of CKD at an early age. Also, various mutations in known inherited diseases such as Polycystic Disease are affecting the course of the disease, with specific genotypes bringing about a much faster development of CKD.

Modified risk factors

- Arterial Hypertension: For patients without proteinuria, the KDIGO guidelines recommend adjusting the blood pressure to levels below $140 / 90 \mathrm{mmHg}$, and if there is proteinuria the target should be lowered to $130 / 80$.

- Proteinuria: Proteinuria and especially at levels above $1 \mathrm{~g} / 24 \mathrm{~h}$ have been associated with progression of kidney disease.

- Diabetes: Diabetes is of epidemic proportions, and its prevalence will double in the next 25 years, particularly in the developing countries. In addition, the explosion in the number of people with diabetes has enormous economic implications. The cost affects individuals and families, as well as health services and also national productivity. The global cost of diabetes was $\$ 150$ billion in 2005 , and will double by 2025 . Diabetes is currently the most common cause of people undergoing Renal Replacement Therapy (RRT) and is responsible for more than $22 \%$ of cases. Over the past decades, the United States Renal Data System (USRDS) figures demonstrate a progressive increase in the number of diabetics entering end-stage renal failure programs. Now, $44 \%$ of all incident patients are diabetic. Approximately, $30 \%$ of patients with diabetic nephropathy eventually progress to end-stage renal failure, and the rest usually die from cardiovascular disease before reaching end stage. Many studies in the last 30 years have shown that strict control of glucosemia levels can delay the progression of CKD, along with other vascular complications of the disease. The latest guidelines recommend a target of $7 \%$ for $\mathrm{Hbalc}^{(7)}$.

- Cardiovascular Disease: CKD is now considered equivalent to cardiovascular risk, but cardiovascular diseases also appear to have a higher risk of developing CKD. The two situations share many predisposing factors such as obesity, metabolic syndrome, dyslipidemias, hyperuricemia, smoking, alcohol and arterial hypertension, diabetes and albuminuria that have already been reported. Among individuals who develop ESRD, the risk of cardiovascular disease is 10 to 20 times 
higher than the general population and increased risks are evident even in mild kidney disease ${ }^{(8)}$.

- Nephrotoxic substances: Overuse of NSAIDs and other analgesics, majority of antibiotics and chemotherapeutics, intravenous contrast agents, use of herbal and other formulations and nutritional supplements that do not have the necessary approvals, exposure to lead and heavy metals.

- Kidney stones/Recurrent Urinary Tract Infections: These are conditions that cause chronic damage and scarring in the renal parenchyma.

- Infectious and other diseases: Patients with neoplasia and chronic infectious diseases such as HIV and viral hepatitis as well as patients exposed to nephrotoxic drugs are at increased risk for CKD.

- Socio-economic situation: There is no evidence of a relationship between the socio-economic situation and the CKD. Social inequalities are likely to affect the health of disadvantaged/vulnerable people for a variety of reasons, including access to educational and health promotion initiatives and the psychological consequences of socio-economic inequalities. This is true for diseases such as hypertension and diabetes in adults, and obesity in children. As these risk factors are also predominant for CKD, it seems likely that socioeconomic factors are also related to CKD. Indicatively, according to data from the UK Kidney Disease Registry, the prevalence of Renal Replacement Therapy (RRT) was higher in socially deprived areas.

CKD is a problem of global public health. The actual incidence and prevalence of CKD is difficult to estimate with certainty, since early to moderate CKD severity is usually asymptomatic. Nevertheless, several important epidemiological approaches in different countries have yielded similar results, estimating the prevalence of the disease at $10 \%$.

In the United States, the prevalence of ESRD increased from about 10,000 in 1973 to 615,899 in 2011. In Europe, the figures are similar to those in the US, with one in ten having some degree of CKD and one in a thousand to have ESRD. Patients with end-stage renal disease consume a disproportionately large share of the state's healthcare resources. The total cost of the ESRD program in the US was approximately $\$ 49,300$ million in 2011 while costs per person per year were over $\$ 75,000$ total, $\$ 32,922$ for transplants and $\$ 87,945$ for dialysis patients ${ }^{(9)}$.

This will place an enormous financial burden on countries, including the cost of the management of end-stage renal failure. The cost of CKD to the English NHS in 2009-10 was estimated at $£ 1.44$ to $£ 1.45$ billion, which was approximately $1.3 \%$ of all NHS spending in that year. More than half this sum was spent on RRT, which was provided for $2 \%$ of the CKD population ${ }^{(10)}$.

Thus, it is medically and economically imperative for awareness, detection, and prevention programs to be introduced across the world, particularly in the developing countries. This will require concerted action from global institutions, governments, health service providers, and medical practitioners.

Despite the recognition that CKD is a progressive disease, its development can be slowed by proper treatment, which is mainly based on: maintaining strict diabetic 
control; lowering blood pressure; healthy eating; low fat salt intake; maximizing lipid control; promoting regular exercise; maintaining normal body weight or reducing weight; smoking and alcohol cessation; avoid nephrotoxic drugs ${ }^{(11)}$. It is a great challenge to communicate the magnitude of the diabetic, cardiovascular, and chronic disease kidney problem to communities and to governments worldwide. Detection and prevention programs, including screening of communities for diabetes, hypertension, and chronic kidney disease have to be implemented in order to disincline this enormous problem of chronic disease. Lifestyle changes, modification of the ill effects of globalization, and massive community education will be needed.

\section{References}

[1] Collins AJ, Foley R, Herzog C, et al. Excerpts from the United States Renal Data System 2007 annual data report. Am J Kidney Dis (2008); 51: S1-320.

[2] White S.L., Cass A, et al: Chronic kidney disease in the general population. Advances in Chronic Kidney Disease (2005); 12(1): 5-13.

[3] Caroline S. Fox, et al: Predictors of New-Onset Kidney Disease in a Community-Based Population. JAMA (2004); 291:844-850.

[4] USRDS 2018 Annual Data Report. Available at https://www.usrds.org/adr.aspx:. Accessed December 9, 2019.

[5] Kazancioğlu R: Risk factors for chronic kidney disease: an update. Kidney Int Suppl (2011). 2013 Dec; 3(4): 368-371.

[6] Iseki K. Factors influencing the development of end-stage renal disease. Clin Exp Nephrol. 2005;9:5-14

[7] Atkins R: The epidemiology of chronic kidney disease. Kidney International, Vol. 67, Supplement 94 (2005), pp. S14-S18.

[8] Elsayed E, et al: Cardiovascular Disease and Subsequent Kidney Disease. Arch Intern Med. 2007; 167:1130-1136.

[9] US Renal Data System 2012 report, http://www.usrds.org/2012/pdf/v2_ch11_12.pdf.

[10] M. Kerr et al: Estimating the financial cost of chronic kidney disease to the NHS in England. Nephrol Dial Transplant (2012) 27 (Supple 3): iii73-iii80.

[11] Drawz P, Rosenberg M: Slowing progression of chronic kidney disease. Kidney Int Suppl (2013); 3: 372-376. 